\title{
Intraluminal irradiation for the palliation of lung cancer with the high dose rate micro-Selectron
}

\author{
Paul A Burt, B Ronan O'Driscoll, H Maeve Notley, Philip V Barber, Ronald Stout
}

\begin{abstract}
Fifty patients with inoperable, symptomatic endobronchial carcinoma were treated by a single exposure of intraluminal radiotherapy. A high dose rate afterloading system (the microSelectron-HDR) was used to minimise radiation exposure for staff. Haemoptysis was relieved in 24 of 28 patients, breathlessness in 21 of 33 patients, and cough in nine of 18 patients. Radiological collapse resolved in 11 of 24 patients. Treatment was given on an outpatient basis and was well tolerated. Intraluminal radiotherapy appears to offer an effective alternative to conventional fractionated external beam radiotherapy.
\end{abstract}

Palliative external beam radiotherapy is an established treatment for patients with nonsmall cell lung cancer who are not suitable for surgery or radical radiotherapy. Although effective in relieving cough, haemoptysis, shortness of breath, chest pain, and dysphagia, there is no evidence that it prolongs life if given to patients who have no symptoms at presentation. ${ }^{1}$ Radiotherapy may be kept in reserve to treat symptoms as they arise as death from metastatic disease may predate the onset of respiratory problems. About 750 patients receive thoracic irradiation each year at the Christie Hospital and Holt Radium Institute. The problem is increasing and represents a large demand on the resources of a busy department.

The idea of endobronchial irradiation is not new. ${ }^{2}$ Unlike external beam radiotherapy, it produces much more localised treatment, sparing the surrounding tissues from the early and late effects of radiation. Various techniques have been used but none has gained widespread acceptance. Iodine-125, gold-198, radon-222, and radium have all been implanted in the bronchus but this method results in poor source distribution and carries a risk of oedema, haemorrhage, or source displacement. Temporary intraluminal irradiation with caesium-137 or iridium-192 is preferred but often means prolonged treatment times and exposure of staff to appreciable radiation doses.

The development of a high dose rate, remote after loading system (using a high activity miniature source) has now overcome the problems associated with endobronchial radiotherapy. A pilot study to examine the effectiveness of intraluminal irradiation has been in progress since April 1988.

\section{Methods}

PATIENTS

Patients included in the study had advanced carcinoma of the bronchus that was producing symptoms likely to respond well to intraluminal irradiation-namely, haemoptysis, dyspnoea, and cough. Chest pain and dysphagia, symptoms due to the mass effect of tumour, were thought unlikely to improve with a localised form of irradiation and patients with these symptoms have continued to receive standard palliative external beam radiotherapy alone. In addition, patients had to be fit enough to undergo flexible bronchoscopy and to have visible endobronchial tumour. Fifty patients were treated during the first six months. Thirty seven were male and 13 female, ranging in age from 43 to 86 (mean 71 ) years. Twenty eight presented with haemoptysis, 33 with breathlessness, and 18 with cough. Histological material was obtained in 41 patients. Thirty six had squamous cell, two large cell, and three small cell carcinoma. The distribution of tumour sites is shown in figure 1 . One patient had bilateral tumours, one affecting the right intermediate and the other the left upper lobe

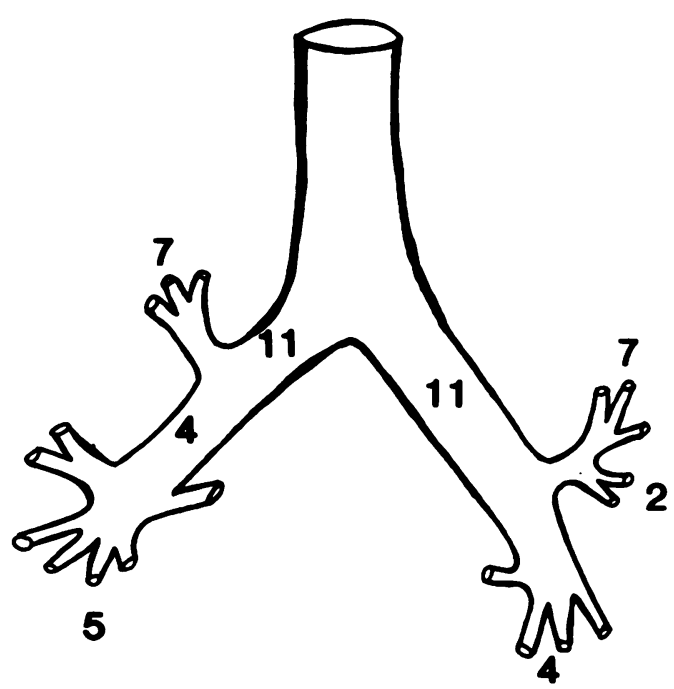

Figure 1 Distribution of tumour sites in the bronchial tree. 
Figure 2 The treatment applicator protruding from the tip of the bronchoscope (centre) and the graduated metal wire insert (below), with a "dummy" inactive iridium source attached to the delivery cable (above).

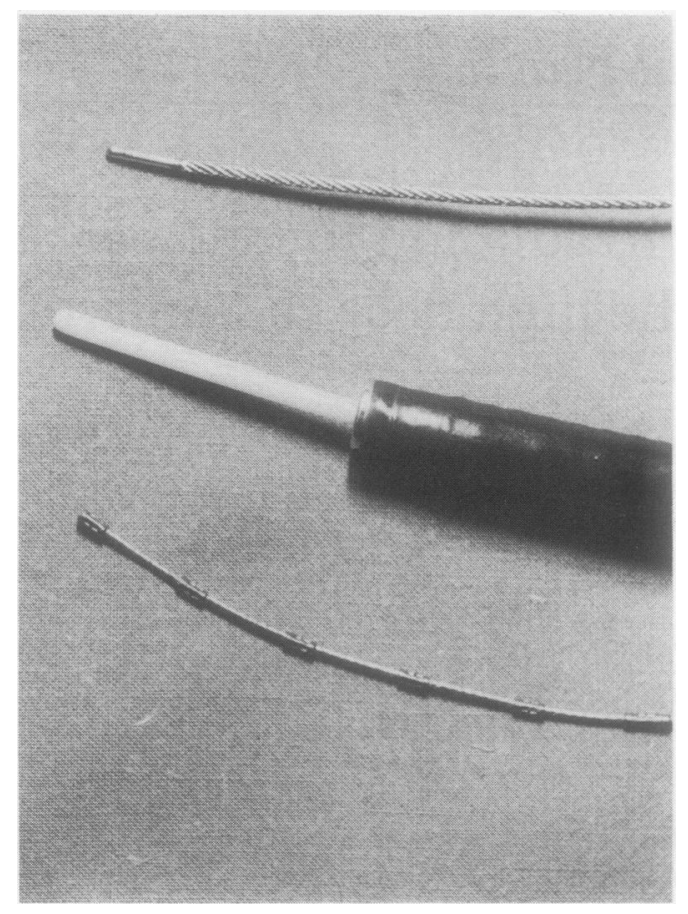

bronchus, and these were treated by means of two applicators placed simultaneously. One patient had received external beam radiotherapy six months before, another had had chemotherapy three months before, and a third laser treatment during the previous month. The remaining 47 were newly diagnosed and untreated.

\section{ASSESSMENT}

A simple subjective assessment of response was made six weeks after radiotherapy by asking the patients if their symptoms were better, the same, or worse. Forty one were also assessed radiologically and 17 had repeat bronchoscopy. Patients were then seen at two monthly intervals. The survivors have a maximum follow up of 10 months and a minimum of four.

\section{IRRADIATION METHOD}

The micro-Selectron-HDR (Nucletron Trading $L t d$ ) is a high dose rate, remote afterloading system using a single, miniature, high activity $(10 \mathrm{Ci})$ iridium-192 source, which is retained within a tungsten safe in the treatment unit when not in use. The source is attached to a cable that is mechanically driven at high speed into $1.9 \mathrm{~mm}$ diameter hollow applicators previously positioned within the patient. A maximum of 18 applicators may be used and the source is guided automatically into each in turn. Treatments are delivered in a shielded room to comply with radiation protection regulations. The micro-Selectron can be installed in an existing radiotherapy facility such as that used for cobalt teletherapy, a linear accelerator, or a brachytherapy theatre. All personnel must leave the room during treatment and patients are observed on closed circuit television. The source can be rapidly withdrawn into the safe in the treatment unit if immediate medical or nursing care is required.

A separate control unit situated outside the treatment room is used to programme the stopping positions and the dwell times for the single source within each applicator. The desired distribution of radiation dose around the tumour is thereby obtained.

\section{PROTOCOL}

Fibreoptic bronchoscopy was performed under local anaesthesia in the $x$ ray room adjacent to the brachytherapy theatre. A large channel bronchoscope was used to identify the site of the tumour and its position relative to the main carina. A hollow treatment applicator was then passed down the working channel of the bronchoscope and positioned alongside or through the endobronchial tumour under direct vision (fig 2). A guide wire, twice the length of the bronchoscope, was inserted into the applicator to facilitate the careful withdrawal of the bronchoscope, leaving the applicator in situ, secured to the nose with tape. The guide wire was then also withdrawn and another metal wire was advanced to the applicator tip. This wire has radio-opaque graduations at $1 \mathrm{~cm}$ intervals over a total length of $27 \mathrm{~cm}$, corresponding to the maximum treatment length that can be programmed with the micro-Selectron (fig 2). Anteroposterior and lateral chest radiographs were taken with this graduated insert in place. The main carina was identified on the plain radiograph and the $1 \mathrm{~cm}$ graduations were used to identify the tumour site and plan the treatment length. The patient was then transferred to the theatre and the applicator connected to the treatment unit. The length of the bronchus to be treated and the source stopping positions within the applicator were calculated on the basis of information gained from the pretreatment radiographs, bronchoscopy, and the localisation films. The iridium source was programmed to advance in $5 \mathrm{~mm}$ steps along the chosen treatment length, and the dwell time in each position was determined by the strength of the source and the prescribed dose. A single exposure of $15-20 \mathrm{~Gy}$ at a $10 \mathrm{~mm}$ radius from the centre of the applicator was given, each treatment lasting 10-25 minutes. At the end of the treatment session the applicator was disconnected from the treatment unit and manually withdrawn.

\section{Results}

Forty six patients were available for the analysis at 6 weeks; four patients had died by this time. Fourteen patients in total have died, one from renal failure, three from cardiovas-

Response assessed at 6 weeks in the 46 surviving patients

\begin{tabular}{lllll}
\hline & & \multicolumn{3}{l}{ Response } \\
\cline { 3 - 5 } & patients & Better & Same & Worse \\
\hline Haemoptysis & 28 & 24 & 4 & 0 \\
Dyspnoea & 33 & 21 & 9 & 3 \\
Cough & 18 & 9 & 9 & 0 \\
Lung collapse & 24 & 11 & 11 & 2 \\
Chest radiograph & 41 & 16 & 11 & 14 \\
Bronchoscopy & 17 & 15 & 2 & 0 \\
\hline
\end{tabular}




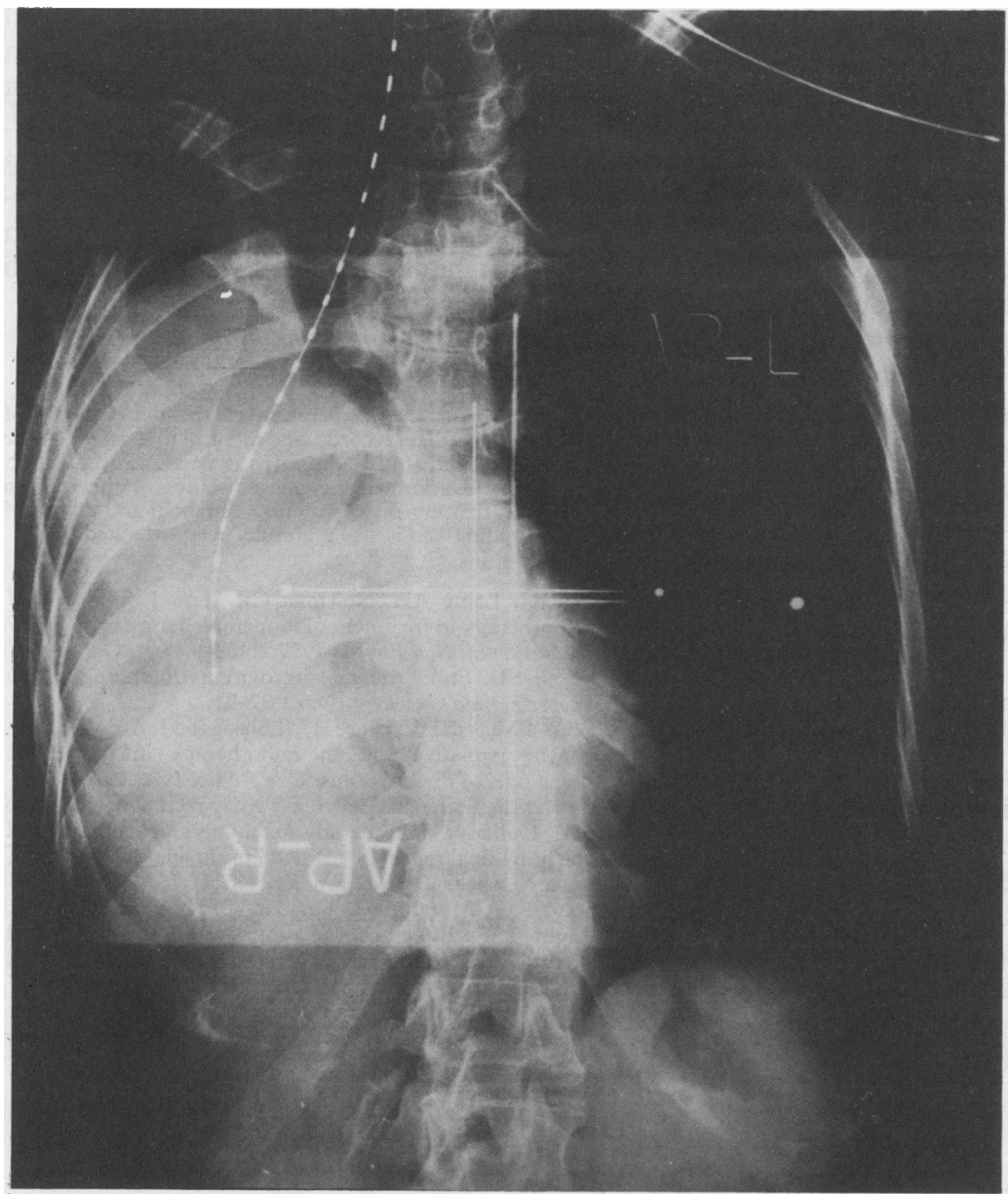

Figure 3 Localisation anteroposterior chest radiograph of a patient with occlusion of the right main bronchus causing complete collapse of the right lung. The treatment applicator with the graduated insert in situ is clearly visible. The distal $10 \mathrm{~cm}$ was programmed for treatment.

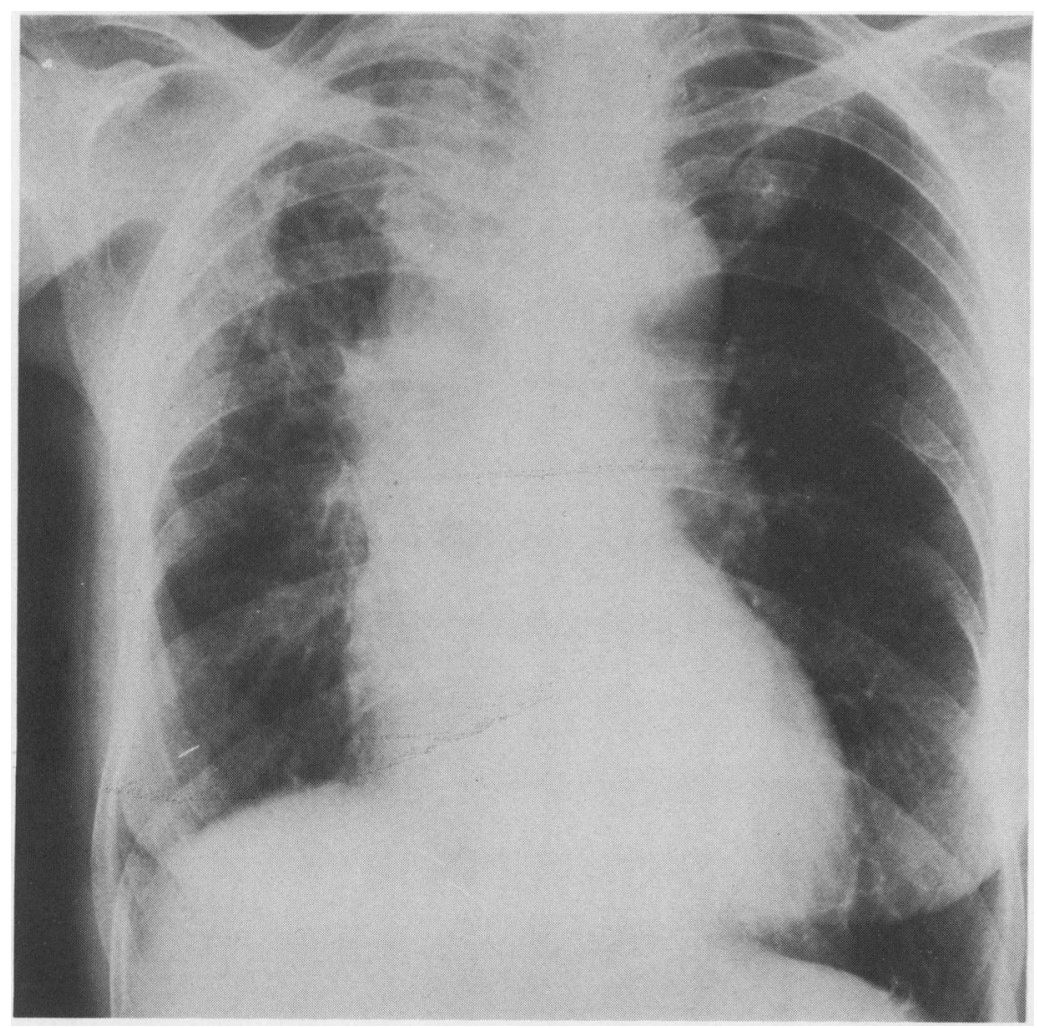

Figure 4 Posteroanterior chest radiograph of the same patient six weeks later showing re-expansion of the lung. cular problems, and the remaining 10 from bronchial carcinoma (median survival 3.5 months). Two died with massive haemoptysis, one at 2 weeks and the other, a non-responder, at 4 months. None of the deaths was considered to be related to treatment.

The procedure was well tolerated. Temporary pleuritic pain was induced in one patient when the applicator was advanced to the pleura and was relieved by its partial withdrawal. No discomfort other than that normally associated with bronchoscopy was encountered. Early morbidity after treatment was limited to one transient exacerbation of tracheal stridor within two hours of treatment and one asymptomatic pneumothorax diagnosed incidentally at the first follow up, six weeks after treatment. Both were managed conservatively. There was no acute radiation oesophagitis and no late morbidity has been observed.

The results of the symptomatic, radiological, and bronchoscopic assessments are shown in the table. Twenty four of the 28 patients with haemoptysis showed substantial improvement and 22 obtained a complete response. The earliest recurrence occurred at 4 months; at that time 19 of the 21 responders still alive were in remission. Haemoptysis has recurred or progressed in three patients, minor in two (at 4 and 6 months) and more severe in one (at 4 months). Only four patients, some five months after intraluminal irradiation, have required external beam radiotherapy for the treatment of troublesome recurrent thoracic symptoms.

\section{Discussion}

Palliative external beam radiotherapy is widely used to relieve symptoms arising from the primary tumour in incurable carcinoma of the bronchus. Haemoptysis, shortness of breath, and cough are caused principally by the endobronchial component and should be improved by treatment capable of clearing the bronchi of macroscopic disease. This study has shown that effective palliation of haemoptysis ( 24 of 28 patients), dyspnoea (21 of 33 patients), and cough (nine of 18 patients) can be achieved with a single exposure of high dose rate intraluminal radiotherapy given under local anaesthesia as a day case procedure. The responses were maintained for at least four months and only four of the 46 surviving patients so far have needed further radiotherapy for the relief of new or recurrent thoracic symptoms. It is a safe treatment with no deaths related to treatment and hardly any morbidity.

Repeat bronchoscopy was performed in 17 patients in the early part of the study. Impressive clearing of tumour was found in 15 , with no residual disease seen at biopsy in many cases. Complete or partial re-expansion of collapsed lung was observed in 11 of 24 patients (figs 3 and 4). Forty one patients had a repeat chest radiograph at their first post-treatment assessment. Although the appearances had deteriorated in 14 , the aims of treatment were achieved in six of these patients as their symp- 
toms had improved with resolution of the endobronchial component of the disease.

These response rates compare very favourably with the results of conventional external beam therapy. The relief of haemoptysis, breathlessness, and cough is similar to that reported with external beam radiotherapy, ${ }^{34}$ whereas re-expansion of collapsed lung was almost twice as good. ${ }^{5}$ The duration of response and survival pattern appear to be similar and these benefits were achieved without the acute oesophagitis and tiredness that occurs in $\mathbf{8 0 \%}$ of patients who have conventional treatment. ${ }^{3}$

Laser resection of endobronchial tumour also produces effective palliation. The relief of symptoms may be quicker but the procedure is certainly more demanding for patient and doctor and associated with more morbidity, and may produce a shorter duration of response. ${ }^{6}$ Intraluminal irradiation and laser resection should perhaps be regarded as complementary rather than alternative treatments. In most cases intraluminal irradiation will suffice but in others, perhaps where there is pulmonary collapse, prior laser resection may enhance the degree of palliation. More experience should enable us to determine which treatment or combination of treatments provides the best palliation with the least morbidity.

The success of this pilot study has encouraged us to start a randomised clinical trial comparing intraluminal radiotherapy with external beam radiotherapy for patients with symptoms due to endobronchial disease alone. A further trial will compare external beam radiotherapy alone with external beam radiotherapy plus intraluminal irradiation in patients who have additional symptoms due to the mass effect of the tumour.

We are grateful for the cooperation of the departments of medical physics, medical records, and medical illustration at the Christie Hospital and Holt Radium Institute in the preparation of this paper and acknowledge the assistance of the radium theatre staff in treating these patients. The help and advice of Nucletron Trading Ltd is also gratefully acknowledged.

1 Berry RJ, Laing AH, Newman CR, Peto J. The role of radiotherapy in treatment of inoperable lung cancer. Int $J$ Radiat Oncol Biol Physics 1977;2:433-9.

2 Seagren SL. Endobronchial irradiation. Endocurietherapy Hyperthermia Oncology 1986;2:87-91.

3 Collins TM, Ash DV, Close HJ, Thorogood J. An evaluation of the palliative role of radiotherapy in inoperable carcinoma of the bronchus. Clin Radiol 1988;39:284-6.

4 Simpson JR, Francis ME, Perez-Tamayo R, Marks RD, Rao DV. Palliative radiotherapy for inoperable carcinoma of the lung: final report of a RTOG multi-institutional trial. Int J Radiat Oncol Biol Physics 1985;11:751-8.

5 Chetty KG, Moran EM, Sassoon CSH, Viravathana T, Light RW. Effect of radiation therapy on bronchial obstruction due to bronchogenic carcinoma. Chest 1989; 95:582-4.

6 Hetzel MR, Nixon C, Edmondstone WM, et al. Laser therapy in 100 tracheobronchial tumours. Thorax 1985;40:341-5. 